\title{
Perspectives in the selection of hyaluronic acid fillers for facial wrinkles and aging skin
}

\author{
This article was published in the following Dove Press journal: \\ Patient Preference and Adherence \\ 2I July 2009 \\ Number of times this article has been viewed
}

\author{
Hannah E John \\ Richard D Price \\ Department of Plastic and \\ Reconstructive Surgery, \\ Addenbrookes Hospital, Cambridge \\ University Teaching Hospitals NHS \\ Trust, Cambridge, UK
}

\begin{abstract}
Aesthetic surgery is, in the USA at least, no longer a taboo subject. Outside North America, public acceptance continues to grow as more procedures are performed each year. While there appears, anecdotally, to be a decrease in patients undergoing cosmetic treatments because of the global financial crisis, the overall trend remains upward. Although popular television programs espouse the benefits of surgery, it is nonsurgical procedures that account, numerically, for the majority of procedures performed; in the USA, there was a 48\% growth from 2000 to 2008 in nonsurgical treatments undertaken by women, and $64 \%$ in men and while the average surgeon might perform 60 blepharoplasty operations in 2007, (s)he would also undertake 375 botulinum injections, and almost 200 filler injections of varying sorts. Clearly there is enthusiasm for nonsurgical treatments, and this trend appears to be rising. With this in mind, we present an overview of the commonest filler injection material, hyaluronic acid. We present the mechanism of action, the purported risks and benefits, and briefly discuss technique.
\end{abstract}

Keywords: hyaluronic acid, filler injection, nonsurgical procedures, technique

\section{Introduction}

Soft tissue facial deformities and skin changes associated with aging are known to affect psychosocial interactions. Aesthetic medicine has changed its focus rapidly over the years as a better understanding of the process of facial aging has affected the treatment approaches. Facial aging is now established to be due to a number of features. These include the involutional loss of dermis, resulting in loss of skin tone, gravitational changes due to loss of elasticity, remodeling of bony and cartilaginous structures, and sun damage causing photo-aging. More recently, the concept of volumetric loss in the face has further added to our understanding; fatty volume both migrates, and is lost from, the face. This occurs in predictable areas and a number of treatments now specifically address this issue either alone, or in combination with traditional rejuvenation techniques.

Some of these factors are preventable - most notably the sun damage to the skin that alters dermal composition - but others are less so; gravitational changes are dependent on the environment and volumetric loss is somewhat unavoidable and largely determined by genetic variables. The overall effect of these processes gives us the features of the aging face; flattening of the forehead, brow, glabella and temporal concavity in the upper face, descent of the nasal tip and flattening of the cheek in the mid face and recession of chin, appearance of jowls, loss of lip fullness, and descent of oral commissures in the lower face. In addition the aging process is manifested in the form of wrinkles throughout the face.
Department of Plastic and Reconstructive Surgery, Addenbrookes Hospital, Cambridge University Teaching Hospitals NHS Trust, Cambridge, UK

Email richard.price@addenbrookes.nhs.uk 
Surgical procedures address laxity within the tissues and reposition the soft tissue of the face, on a 'macro' scale. Additional 'micro' adjustment can be made with fat transfer at the time of surgery, the so-called 'volumetric' facelift. Surgical fat transfer performed independently is still useful but does involve a hospital or clinic stay, and is quite clearly an invasive procedure. Importantly, however, surgical fat transfer, while elegant, does not address the finest lines in the face.

Wrinkles caused by active muscle contraction can be ameliorated by the use of botulinum toxin. For established lines there is anecdotal evidence that repeated use of this modality may allow the reversal of superficial lines, although formalized studies into this are limited. There is also a trend amongst the younger population to undergo botulinum treatments in order to prevent the formation of wrinkles, particularly in the forehead area. That said, most treatments for established wrinkles are aimed at volumetric replacement of soft tissue: dermal and/or deep tissue plane augmentation.

The ideal filler substance should be nonallergenic, noncarcinogenic, with minimal adverse sequelae, no associated migration and minimal inflammatory response. It should be reproducible, durable, simple and painless to administer, with minimal recovery time, user-friendly, easily stored and large amounts should be readily available. It should preferably have a long-lasting effect with slow degradation in the body. There are advantages to temporary (rather than permanent) fillers; if the result is not to the patients liking, then it will be reversed over a period of time. Permanent fillers, while an attractive proposition, have two obvious drawbacks; misplacement of filler usually necessitates surgical removal, and the filler placement cannot subsequently be adjusted to account for ongoing age-related changes in the face.

\section{History of soft tissue augmentation}

The first surgical soft tissue augmentation agent used was autologous fat transplantation done by Neuber in $1893 .{ }^{1}$ Paraffin was then tried but was seen to be associated with severe granuloma formation. Subsequently pure silicone was tried but this resulted in severe facial distortion with time. The popular use of dermal bulking agents however, started with the use of injectable bovine collagen in the early 1980s. As a small percentage of patients demonstrated allergy to this product, test injections were necessary, up to three weeks in advance of the actual procedure. More recent alternatives include acellular human dermis (Dermalogen ${ }^{\circledR}$ ) and cultured fibroblasts $\left(\right.$ Isologen ${ }^{\circledR}$ ); these were associated with less immunological intolerance and longer duration of action.
The history of medicine and, particularly aesthetics, is littered with ill-conceived ideas and treatments that might, at best, be described as 'experimental', poorly researched, and launched on an unsuspecting public with variable results. Most historical treatments have survived the test of time and therefore persist, with studies into efficacy retrospective rather than prospective. Unusually within this field, hyaluronic acid is a molecule that has been thoroughly researched and developed, initially for use in ophthalmic surgery, and then unsuccessfully trialed in orthopedic surgery, it came to the attention of plastic surgeons through the scientific field of tissue engineering. Within this latter field it was extensively researched with a view to producing a number of materials, in particular, dermal regeneration templates for use in reconstructive surgery (Figure 1). There are a number of formal research programs dedicated to the material, with significant international collaboration.

\section{Biology of hyaluronic acid}

Chemically, hyaluronic acid (Hyaluronan; HA) is a linear polymer of dimeric $\mathrm{N}$-acetyl glucosamine and glucuronic acid (Figure 2) in huge macromolecular chains., ${ }^{2,3}$ The biology (mainly with regard to tissue engineering) has been extensively reviewed by the senior author ${ }^{3}$ but, for summary purposes, a resume is given here.

Hyaluronic acid is naturally present in the skin as part of the extracellular matrix and as a component of collagen. Its chemical structure is extremely well preserved across a number of species from Ascaris slime to mammals, making allergic reactions very rare. Due to its viscoelastic properties it acts as a ground substance of the dermis, and is also a component of joint fluid, vitreous of eye, disc nucleus, and the umbilical cord. It performs functions such as space

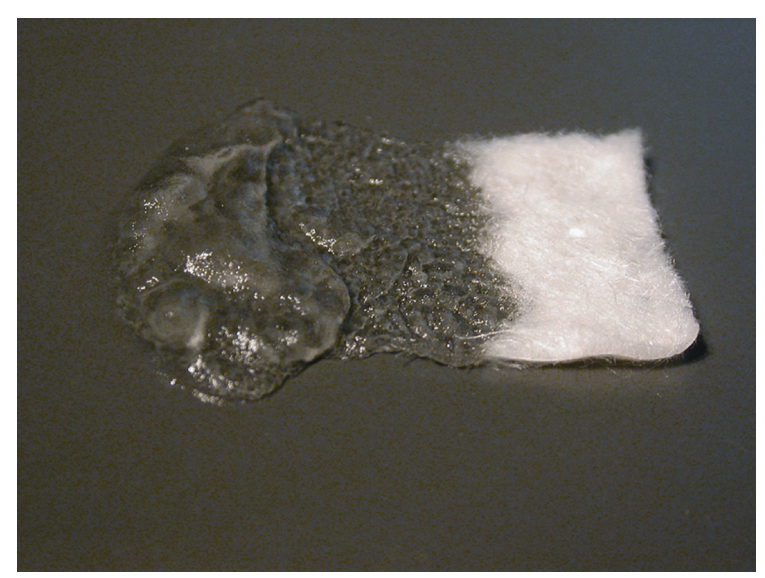

Figure I Tissue engineering substrate made of hyaluronic acid. Water has been applied to one end of this sheet in order to demonstrate the solubility of the material. 


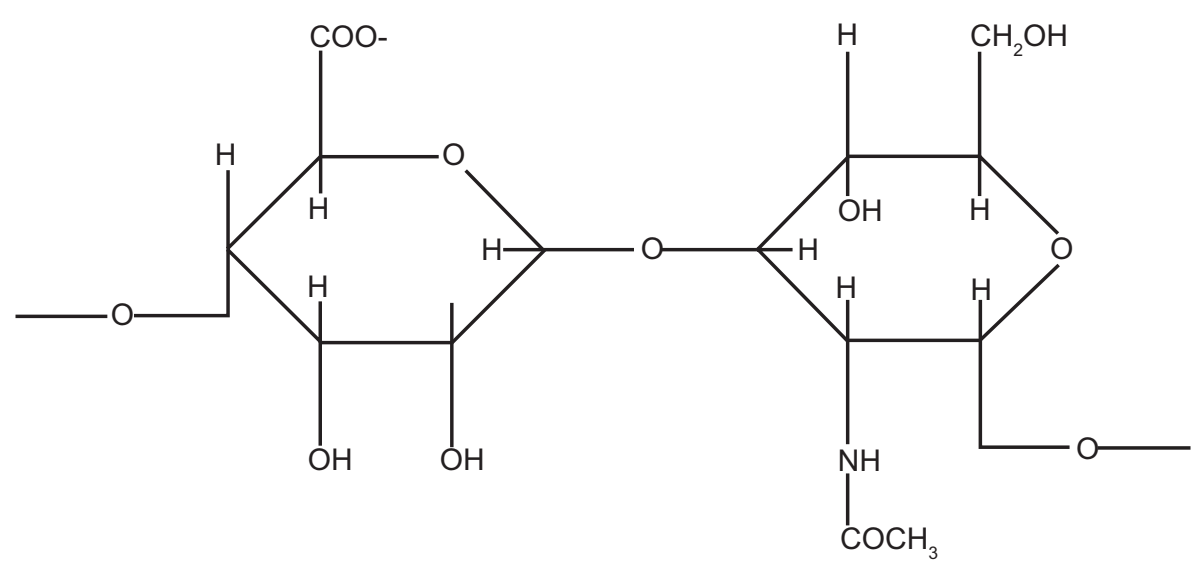

Figure 2 The chemical composition of hyaluronic acid. This diamer is polymerized into macromolecular chains which are then stabilized.

filling, lubrication, shock absorption, and also modulation of inflammatory cells and scavenging of free radicals.

Hyaluronic acid is extremely hydrophilic and biochemically retains water: hydrogen bonding occurs between adjacent carboxyl and $\mathrm{N}$-acetyl groups to the extent that it retains up to 1000 times its weight in water. Sutherland and colleagues demonstrated that one gram of HA can bind up to 6 litres of water. ${ }^{4}$ In the skin, levels of HA naturally decrease with each passing year, resulting in dermal dehydration and rhytide formation (wrinkles), a process accelerated by free radicals.

Hyaluronic acid was first isolated in bovine vitreous by Meyer and Palmer in 1934. ${ }^{5}$ Therapeutic use of various forms of hyaluronic acid started in ophthalmic surgery and later in orthopaedic surgery as a lubricant for joints, in patients with osteoarthritis. ${ }^{6}$ Native HA was noted to last only for a couple of days, but cross linking and chemically modifying (by esterification) the molecule fortified it against enzymatic degradation and improved its longevity and biocompatibility. Furthermore, the degree of esterification and cross-linking can be adjusted to alter the viscosity of the product, an important feature that has served to expand the range of materials available for cosmetic use.

The duration of the cosmetic effect is determined primarily by enzymatic degradation by fibroblasts, resulting in the formation of shorter HA chains, which are then ingested by fibroblasts, macrophages and keratinocytes. From a tissue engineering perspective, these short-chain molecules have a number of specific wound healing properties in themselves, although this falls outwith the remit of this article.

\section{Manufacture}

Hyaluronic acid is identical in structure in all vertebrates but there are differences in protein and nucleic acid contaminants.
There is no antigenic specificity for tissue or species and hence it has a low potential for allergic or immunogenic reactions. The various sources of HA include bacteria (Streptococcus zooepidemicus), rooster comb, human umbilical cord, and bovine vitreous humor. Adverse reaction to different preparations of HA has been established to be due to the cross linking process used rather than the source or nucleic acid contaminants, though the exact reason for the reactions is still unclear. ${ }^{7}$ The viscoelastic property of hyaluronic acid is determined by the length of the molecular chain, the concentration, the cross linking and the particle size. The differences in the various HA agents are decided by source derivation (animal vs. bacteria), cross linking (the method used to create cross linking and the degree of cross linking present), the concentration of HA, the amount of free HA (noncross-linked) and the particle size and uniformity of structure. Hence different manufacturing processes have different gel hardness and different amount of swelling caused by accumulation of water.

As a result of the (controlled) variability in manufacturing processes, a number of products are now commercially available. Generally, these are most easily conceived of with relation to the viscosity of the product and, therefore, the locations for which they would be indicated. For example, a viscous filler with a larger 'granule' size would not be suitable for injecting into the lips because of palpability (Figure 3). A softer product would not give the volume enhancement needed in deep tissue planes, etc. It is for this reason that the practitioner should have at hand a range of products so that bespoke treatment may be offered.

The primary indications for HA fillers are photo-aging wrinkles and in the nasolabial folds, lip rhytides, marionette lines, and volume enhancement such as lip filling and 
A

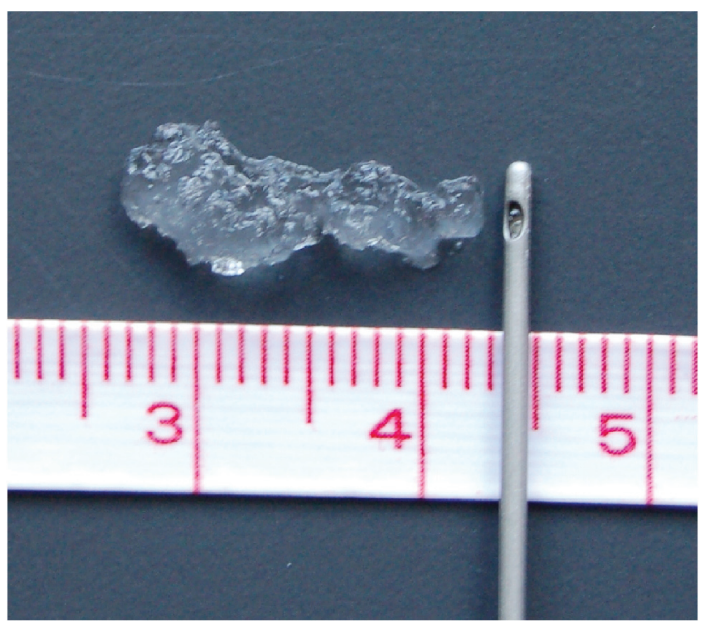

B

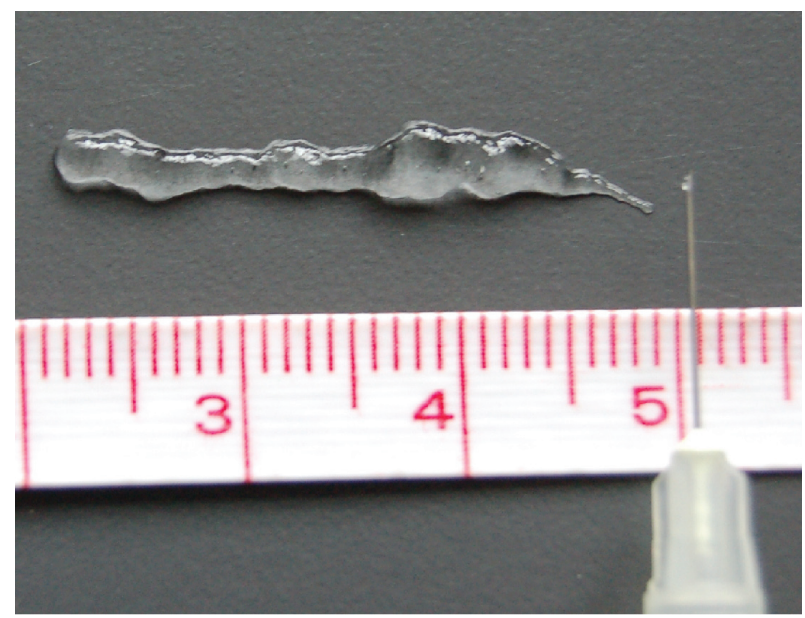

Figure 3 (A) Restylane SubQ ${ }^{\circledR}$, a large-granule product, for deep dermal placement. (B) Juvederm Ultra $2^{\circledR}$, a finer product for more superficial use. Note the difference in texture and the needles used for insertion; the former is placed using a cannula, whilst the latter is injected using a fine-gauge needle.

contouring, chin and cheek augmentation, and treatment of tear trough lines. Generally HA is not ideal for superficial wrinkles, instead it is more suited for deep folds and volume augmentation. ${ }^{8}$ But smaller molecular preparations have been shown to have good effect with superficial wrinkles. After a full pre-treatment assessment and pre-treatment photographs, the correct product should be selected. The greater the viscosity of the gel, the better the ability to resist shear and it can better exert a deformational force on surrounding tissue to correct a defect. The pay-off for such qualities is palpability and firmness to touch. The more superficial the injection, the higher the risk of visibility. This led to various HA products being used for different parts of the face due to difference in residence time, persistence, injectability, and need for local anesthetic.

\section{Method for injection of hyaluronic acid}

The procedure is generally carried out in the outpatient clinic setting, with the patient in an upright position for gravitational wrinkles to be visible, with the head supported on a head rest to avoid sudden movements. Patients on aspirin or anticoagulants are advised to stop these beforehand. Anesthesia, where applied, is usually in the form of topical anesthetic but field blocks or nerve blocks may be required especially for lip procedures which can otherwise be quite painful.

Using a 27-30 gauge needle, the agent is injected into the middle to deep dermis, except in lips where it is injected intramuscularly rather than intradermally. Larger molecular HA will require larger gauge needles for injection. If injected subcutaneously the material is wasted as its bioavailability is very short due to quicker enzymatic degradation and the effect may be limited. There are five commonly used techniques:

1. Linear threading technique: the fold is filled by a single puncture to the epidermis and injecting the HA agent along the track of the needle producing linear volume. This is the commonest technique used by the senior author.

2. Serial puncture technique: the fold is filled by multiple injections of the agent, all in a row.

3. Fanning technique: here the epidermis is punctured once, and then the needle is fanned out while injecting the agent, producing volume in a triangular shape.

4. Cross-hatching technique: this combines multiple injections of linear threading that crisscross at right angles to provide volume in a square shape. This is analogous to fat transfer techniques and is, in the author's opinion, the best technique for deeper volumetric adjustment.

5. Fern pattern technique: This involves serial puncturing in a linear manner but is done perpendicular to the actual fold on either side of it, increasing dermal stiffness. ${ }^{9}$

The senior authors' current practice involves using a layering technique where heavier gels are injected into the deeper layers and lighter gels into the superficial layers. Constant movement of the needle is important in producing an even distribution of product, and avoiding injecting into a blood vessel. Injection just above the periosteum is very favorable as it produces re-inflation of the natural fat pads, in the brow, malar, buccal, and mental areas. After injection, the area should be massaged to smooth out any minor irregularities but care should be taken not to migrate the filler into an undesirable location. An ice pack may be used 
to decrease swelling and pain and the patient is advised to minimize movement of the injected area and avoid anticoagulants for the next couple of days to reduce incidence of bruising. Some physicians also advise abstinence from alcohol in the immediate post-procedure period, but evidence to support this is limited.

Combining HA filler treatment with injection of botulinum toxin is increasingly popular because it extends the cosmetic response of the HA agent and immobilizing the muscles increases the biodegradation time. ${ }^{10}$ However, research is limited on the subject of concommitant injection and the senior author prefers to separate botulinum and filler treatments by at least two weeks, performing the former first. Depending on the filler agent used and the depth at which it is injected, the effects last for 4-12 months.

It is difficult to be specific on anticipated duration of effect and patients should be advised of this. However, a few guidelines may be reasonably quoted. Most injections last at least three months and often up to six months, although there are anecdotal reports of longer duration and, indeed, some of the newer products claim up to 12 months efficacy. On balance, it is probably best to advise patients to expect 4-6 months efficacy, and that treatments may be needed 2-3 times per annum. Concommitant treatment with botulinum toxin may prolong these effects. ${ }^{11}$

There is some suggestion, although not proven as yet, that the repeated application of HA in the same area does, in fact, eventually provide a long-lasting result; whether this is due to the sustained increase of extracellular matrix, or whether this is the result of repeated scarring is not known.

However it is to be noted that intertreatment times are dependent upon patients representing; there is therefore a number of biases including patient perception of the effect, financial considerations, and the fact that this population are notoriously fickle. The senior author has a number of patients who claimed to be happy with their previous surgeon, but still chose to move to his practice for a variety of reasons unrelated to their previous practitioner.

\section{Adverse reactions}

Adverse reactions associated with HA fillers include bruising, edema, erythema, irregularities secondary to nodule formation, and delayed inflammatory reactions. These are seen in up to $12 \%$ of patients. ${ }^{12}$ The incidence of these reactions is much less frequent compared to injectable collagens and the volume of HA agent required is significantly less than that of collagen. Side effects such as sterile abscesses, ${ }^{13}$ induction of sarcoid, ${ }^{14}$ and even angioedema ${ }^{15}$ have also been reported. Significant complications such as hematoma formation, significant swelling, etc are reported to occur in up to $1: 1600$ cases. $^{13}$

\section{Topical hyaluronic acid}

Topical HA is not cross linked and is therefore easily absorbed. It was first used as a vehicle for the delivery of other drugs to the skin, particularly useful for sustained release and localized delivery and passes through the skin by active transport. ${ }^{9}$ As the significance of HA in water retention in the dermis has come to light, topical pure HA has become a cosmetic in its own right. In the superficial epidermis it acts as a humectant contributing to moisture content, and it decreases transepidermal water loss. ${ }^{16}$ Once absorbed into deeper dermis, it increases turgidity within the dermis. It also assists in proliferation of dermal fibroblasts, promoting extracellular matrix production in vitro, ${ }^{17}$ although there is no clear evidence this occurs in vivo. Since noncross-linked HA has a short half life, this will need frequent application, but as it may increase the water retention of the dermis, it is marketed as a plumping agent for the skin. The authors are not aware of any objective studies in this area and cannot comment upon the claims made.

\section{Disclosure}

The authors report no conflicts of interest in this work.

\section{References}

1. Fagien S, Klein A. A brief overview and history of temporary fillers; evolution, advantages and limitations. Plast Reconstr Surg. 2007;120(6 supp1):8S-16S.

2. Campoccia D, Hunt JA, Doherty PJ, et al. Quantitative assessment of the tissue response to films of hyaluronan esters. Biomaterials. 1996;17:963-975.

3. Price RD, Berry MG, Navsaria HA. Hyaluronic acid: the scientific and clinical evidence. J Plast Reconstr Aesthet Surg. 2007;60: 1110-1119.

4. Sutherland I. Novel and established applications of microbial polysaccharides. Trends Biotechnol. 1988;16:41-46.

5. Meyer K, Palmer JW. The polysaccharide of the vitreous humor. J Biol Chem. 1934;107:629.

6. Petrella RJ, DiSilvestro D, Hildebrand C. Effects of hyaluronate sodium on pain and physical functioning in osteoarthritis of the knee: a randomised double blind placebo controlled clinical trial. Arch Intern Med. 2002;162:292-298.

7. Goldberg VM, Coutts RD. Pseudoseptic reactions to hylan viscosupplementation: Diagnosis and treatment. Clin Orthop Relat Res. 2004;419:130-137.

8. Monheit GD, Coleman KM. Hyaluronic acid fillers. Dermatol Ther 2006;19:141-150.

9. Van Eijk T, Braun M. A novel method to inject hyaluronic acid: the fern pattern technique. J Drugs Dermatol. 2007;6(8):805-808.

10. Carruthers J, Carruthers A, Maberley D. Deep resting rhytides respond to BTX-A and Hylan-B. Dermatol Surg. 2003;29(5):539-544. 
11. Rohrich RJ, Ghavami A, Crosby MA. The role of hyaluronic acid fillers (Restylane) in facial cosmetic surgery: review and technical considerations. Plast Reconstr Surg. 2007;120(6 Suppl):41S-54S.

12. Friedman PM, Mafong EA, Kauvar AN, Geronemus RG. Safety data of injectable non-animal stabilized hyaluronic acid gel for soft tissue augmentation. Dermatol Surg. 2002;28:491-494.

13. Leonhardt JM, Lawrence N, Narins RS. Angioedema acute hypersensitivity reaction to injectable hyaluronic acid. Dermatol Surg. 2005;31:577-579.

14. Dal Sacco D, Cozzani E, Parodi A, Rebora A. Scar sarcoidosis after hyaluronic acid injection. Int J Dermatol. 2005;44:411-412.
15. Lowe NJ, Maxwell CA, Patnaik R. Adverse reactions to dermal fillers: review. Dermatol Surg. 2005;31:1616-1625.

16. Brown TJ, Alcorn D, Fraser RE. Absorption of hyaluronan applied to the surface of intact skin. J Invest Dermatol. 1999;113:740-746.

17. Weindel G, Schaller M, Schäfer-Korting M, Korting HC. Hyaluronic acid in the treatment and prevention of skin diseases: molecular biological, pharmaceutical and clinical aspects. Skin Pharmacol Physiol. 2004;17(5):207-213.

18. Greco R, Iocono J, Ehrlich P. Hyaluronic acid stimulates human fibroblast proliferation with a collagen matrix. J Cell Physiol. 1998;177:473-475.

\section{Publish your work in this journal}

Patient Preference and Adherence is an international, peer-reviewed, open access journal that focusing on the growing importance of patient preference and adherence throughout the therapeutic continuum. Patient satisfaction, acceptability, quality of life, compliance, persistence and their role in developing new therapeutic modalities and compounds to optimize clinical outcomes for existing disease states are major areas of interest. This journal has been accepted for indexing on PubMed Central. The manuscript management system is completely online and includes a very quick and fair peer-review system. Visit http://www.dovepress.com/ testimonials.php to read real quotes from published authors. 